\title{
A proposito di due cassette per viscere di età tolemaica nel Museo Egizio di Torino (Cat. 2423 e Cat. 2425)
}

\section{Paolo Marini}

Two wooden, plastered and painted chests for viscera, belonging to Tabes (Cat. 2423) and Tasheritnetkhonsu (Cat. 2425), are held at the Museo Egizio in Turin. Produced between the end of the Late Period and the beginning of the Ptolemaic Period, they have never been studied in depth. The aim of this paper is to provide detailed morphological and iconographic descriptions of these chests, to present a transliteration and translation of the hieroglyphic texts on them, to discuss their date through stylistic comparisons and a prosopographical study, and to draw some conclusions about their true function and, more generally, about the function of this kind of objects. The author argues that both chests come from Bernardino Drovetti's collection, acquired by the Museo Egizio in 1824. They were probably found in Ptolemaic burials within a Late Period Theban Tomb (TT 414), investigated by Drovetti's agents in the early nineteenth century.

\section{ملخص البحث:}

Cat. ) يوجد بالمتحف المصري بتورينو صندوقان خشبيان تغطيهم طبقة ملونة من الجص لحفظ الأحشاء وينتميان إلى تا-بس 2423)، تا-شريت-نat. 2425) (تمنست صنع هذين الصندوقين في الفترة ما بين نهاية العصر المتأخر وبداية العصر البطلمي، ولم يتم دراستهما بتعمّق.

تهذف هذه الدراسة إلى توفير وصف بنيوي وتصويري مفصل لهذان الصندوقان، وتقديم اللفظ الحرفي للنصوص الهيروغليفية المنقوشة عليهما وترجمتها، بالإضافة إلى مناقشة تاريخهما من خلال إجراء مقارنات أسلوبية ودراسة الخصائص المشتركة التاريخية لمن إستخدموا هذا النوع من الأدوات، بالإضافة إلى إستخلاص بعض الإستتتاجات حول وظيفتهما الحقيقية وجدوى هذا النوع من الصناديق بشكل عام.

يرى المؤلف أن كلّ الصندوقين ينتميان إلى مجموعة "برناردينو دروفيتي"، التي حصل عليها المتحف المصري في عام 1824. كما يُعتقد بأنهما قد تم إكتثافهما على الأرجح ضمن مقابر العصر البطلمي داخل مقابر طيبة الجنائزية الخاصة بالعصر الفرعوني المتأخر (TT 414) التي قامت بتتقيبها بعثة دروفيتي في أوائل القرن التاسع عشر.

Al Museo Egizio di Torino ${ }^{1}$ si conservano due piccole "cassette funerarie", inventariate con il numero Cat. 2423 e Cat. 2425 (Fig. 1), che non sono mai state oggetto di uno studio approfondito. Pier Camillo Orcurti $^{2}$ ne fornisce una prima sintetica descrizione alle voci n. 233 e 235 del Catalogo illustrato dei monumenti egizii del R. Museo di Torino, ${ }^{3}$ ripresa da Ariodante Fabretti, Francesco Rossi e Ridolfo Vittorio Lanzone, che le includono nel catalogo del Regio Museo di Torino ${ }^{4}$ con i numeri 2423 e 2425, senza tuttavia specificarne mai la funzione.

Nessun documento d'archivio noto ne racconta le vicende collezionistiche moderne, ${ }^{5}$ ma la presenza nel catalogo dell'Orcurti costituisce un terminus ante quem che ne suggerisce l'arrivo a Torino con la collezione venduta dal Console Generale di Francia ad Alessandria, Bernardino Drovetti. ${ }^{6}$ Tale ipotesi pare supportata dai dati esposti a seguire. Infatti alcune considerazioni prosopografiche, insieme alla datazione e a una comparazione iconografica, spingono 


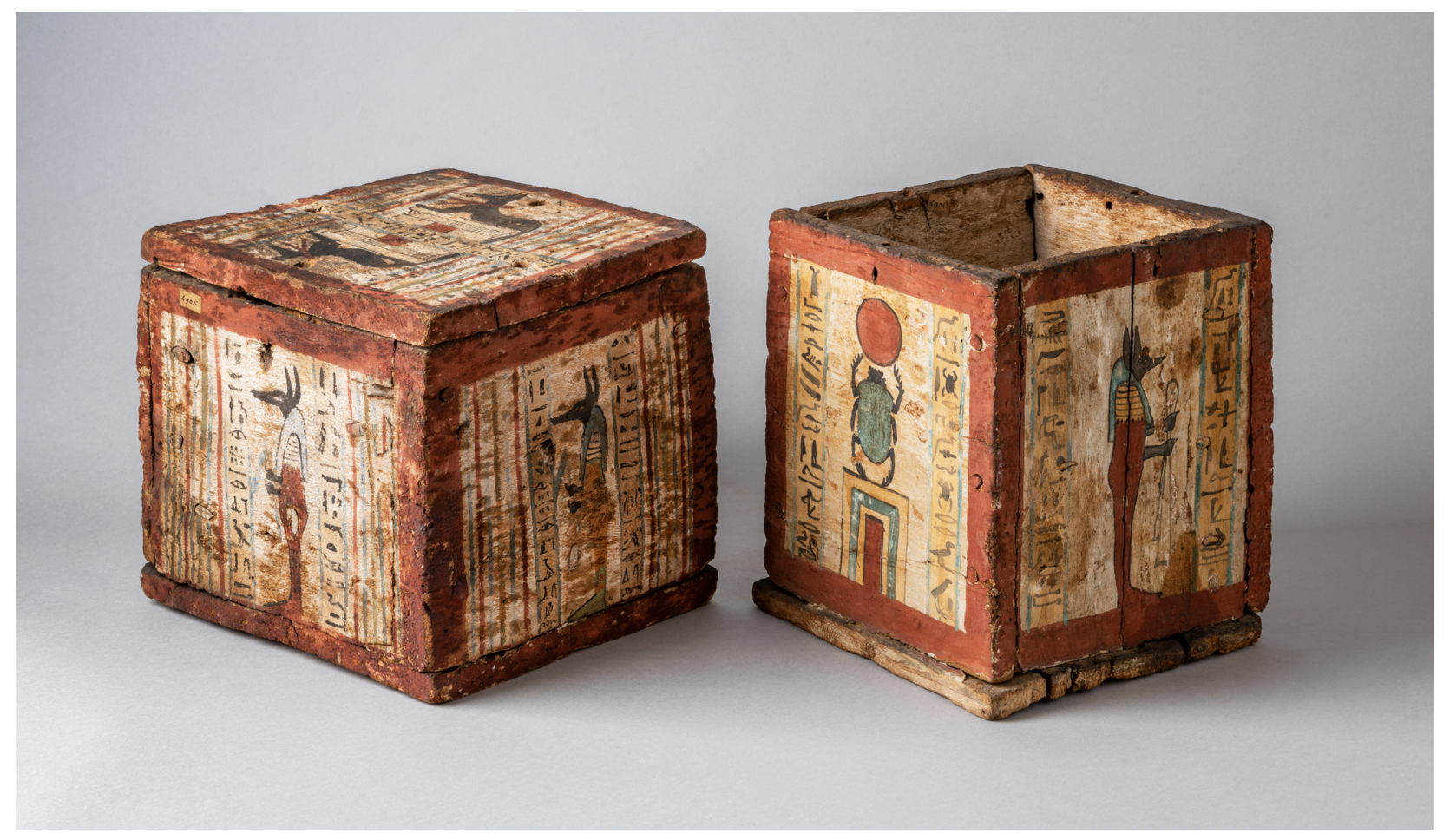

Fig. 1: Cassette di Tabes (Torino Cat. 2423) e Tasheritnetkhonsu (Torino Cat. 2425). H. 22,3 e 24,5 cm. Foto di Federico Taverni/Museo Egizio, Torino.

a collocare il loro ritrovamento nella necropoli tebana, presso un contesto di scavo indagato dagli agenti del Console.

\section{Descrizione \\ 1.1 Torino Cat. 2423}

(Tav. 1-3)

La cassetta lignea Torino Cat. 2423 è composta da un contenitore pressoché cubico, di modeste dimensioni (22,3 x $24 \times 22,5 \mathrm{~cm})$, e dal suo coperchio piatto. Le pareti laterali e il fondo sono ricavati da singoli pezzi di legno, assemblati mediante l'uso di cavicchi troncoconici disposti diagonalmente lungo gli spigoli. Il coperchio è caratterizzato da quattro fori laterali che lasciano presupporre il medesimo uso di cavicchi per ancorarlo al corpo principale del contenitore. Sulla superficie interna sono presenti tracce del sottile strato di stucco che la doveva interamente ricoprire, mentre su quella esterna tale strato si conserva quasi integralmente, malgrado delle piccole lacune. Molto probabilmente, lo stucco è stato applicato una volta assemblate le cinque facce del corpo principale, in modo da nascondere le giunture e regalare all'oggetto una superficie uniforme. Infine sono stati applicati i colori, realizzando un programma decorativo molto semplice e dalla resa tecnica mediocre.

Sulle quattro pareti verticali della cassetta, su un fondo bianco, si ripete la rappresentazione del dio Anubi raffigurato con il corpo mummificato - in due casi di colore rosso e in due verde - il petto impreziosito da un collare usekh giallo con filari neri, la testa di sciacallo nera e una parrucca tripartita di colore blu. La divinità è rappresentata stante, nell'atto di impugnare con entrambe le mani un fazzoletto ripiegato, tra due colonne di geroglifici neri su fondo giallo, bordate da linee di colore blu. Esternamente alle colonne di testo vi è un motivo decorativo composto da linee verticali rosse, blu e verdi. Lungo gli spigoli di ognuna delle quattro pareti corre una banda di colore rosso che incornicia ogni singola scena. La parete esterna del coperchio reca al centro una colonna di geroglifici neri su fondo giallo, bordata da linee blu, che funge da base per la rappresentazione speculare di due sciacalli neri, accovacciati su un padiglione bianco sormontato da una cornice a gola. I due canidi sono affiancati - davanti e dietro - da una serie di linee parallele rosse, blu e verdi. Come per le pareti, lungo i bordi corre una banda rossa che incornicia la scena (Tav. 3).

Le iscrizioni geroglifiche delle colonne recano il seguente testo: 

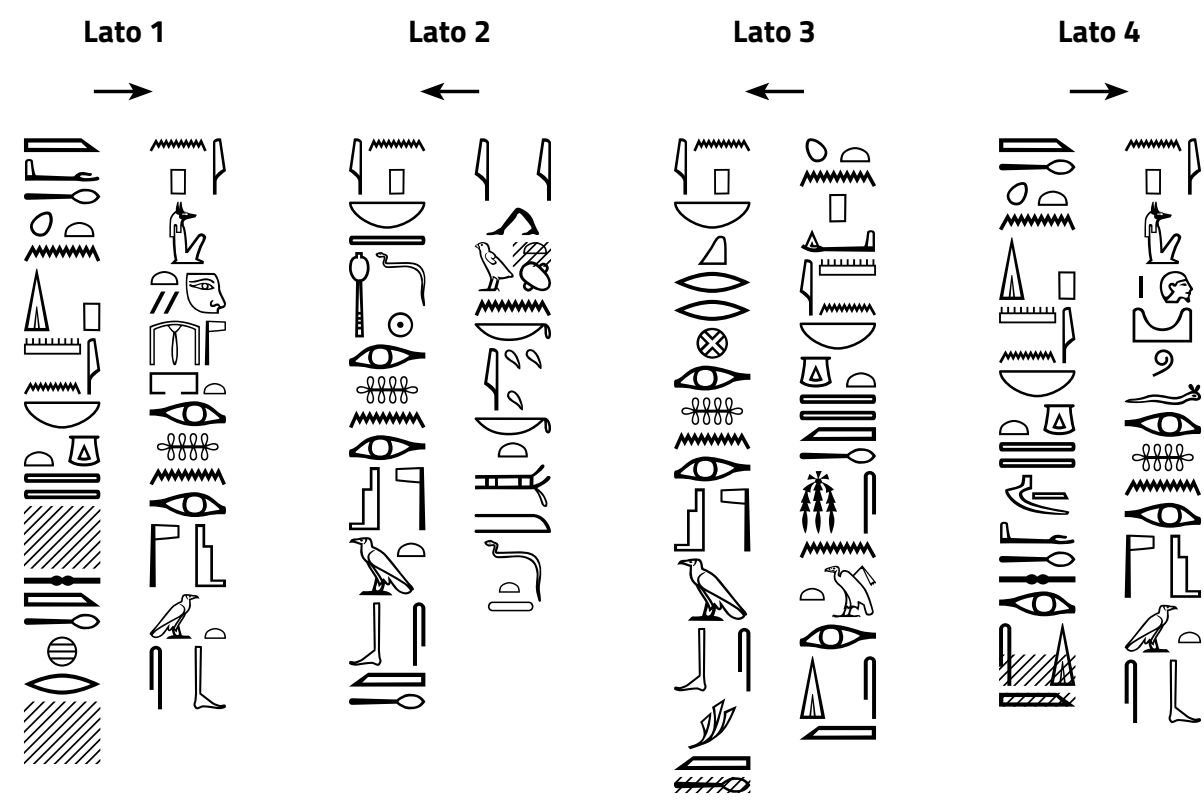

Coperchio

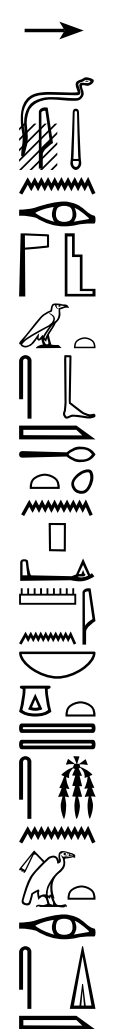

Lato 1:

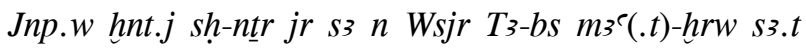
$n$ (.t) P3-dj-Jmn-nb-ns.wt-t3.wj m3-hrrw hrr [ntrr c3?]

Anubi, colui che presiede il padiglione divino, colui che fa la protezione per l'Osiri Tabes, giusta di voce, figlia di Padiamunnebnesuttauy, giusto di voce presso [il grande dio?]

\section{Lato 2:}

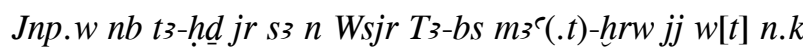
$j w f=k t m \underline{d} t$

Anubi, signore di Ta-hedj (Gebelein), ${ }^{7}$ colui che fa la protezione dell'Osiri Tabes, giusta di voce. Che venga l'imbalsamatore per te, affinché la tua carne sia tutta (completa, integra) per l'eternità.

\section{Lato 3:}

Jnp.w nb krr(.t) jr s3 $n$ Wsjr T3-bs m3e(.t)-hrw s3.t $n(. t)$

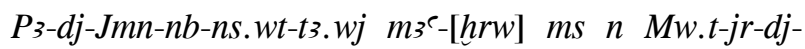

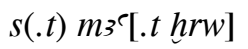

Anubi, signore della caverna, ${ }^{8}$ colui che fa la protezione dell'Osiri Tabes, giusta di voce, figlia di Padiamunnebnesuttauy, giusto di voce; nata da Mutirdis, giusta [di voce].
Lato 4:

Jnp.w tp(.y) $\underline{d} w=f$ jr s3 $n$ Wsjr T3-bs m3e(.t)-hrrw s3.t $n$ (.t) P3-dj-Jmn-nb-ns.wt-t3.wj $m_{3}{ }^{e}-h r w<m>s<n$ $M w . t>[j r]-d j-s(. t) m_{3}{ }^{\complement}[. t-h r w]$

Anubi, colui che è sulla sua montagna, colui che fa la protezione dell'Osiri Tabes, giusta di voce, figlia di Padiamunnebnesuttauy, giusto di voce; <nata da Mut>irdis, giusta [di voce]. ${ }^{9}$

\section{Coperchio:}

dd md.w jn Wsjr T3-bs m3e(.t)-hrww s3.t n(.t) P3-dj-jmn$n b-n s . w t-t 3 . w j$ ms $n M w . t-j r d j-s(. t) m_{3}{ }^{-}[. t-h r w]$

Parole dette dall'Osiri Tabes, giustificata, figlia di Padiamunnebnesuttauy; nata da Mutirdis, giusta [di voce].

\subsection{Torino Cat. 2425}

\section{(Tav. 4-5)}

La cassetta lignea Torino Cat. 2425 è un contenitore di forma pressoché cubica, di dimensioni modeste $(24,5 \times 20,3 \times 20,6 \mathrm{~cm})$. Le quattro pareti sono ancorate ad una base sporgente mediante dei cavicchi troncoconici ancora in situ. La presenza di quattro fori trasversali sullo spessore superiore delle pareti lascia presupporre l'originaria presenza di un coperchio oggi perduto. Un sottile strato di stucco con 
pittura policroma ricopre interamente la superficie esterna e reca un articolato programma decorativo, sebbene di qualità mediocre. Al centro di uno dei lati è rappresentata una cappella del tipo $p r-n w$ (santuario del Basso Egitto), ${ }^{10}$ di colore giallo, dalla quale fuoriesce la testa del dio Sokar raffigurato con una parrucca corta sormontata da un disco solare disposto tra le due corna di ariete e la doppia piuma. Ai lati di questa immagine corrono due colonne di geroglifici neri su fondo giallo, bordate da linee di colore blu; sul lato opposto è rappresentata una struttura architettonica dal profilo rettangolare, decorata con il motivo a "falsa-porta" in giallo, blu e rosso. La struttura è sormontata dal dio Khepri rappresentato come uno scarabeo di colore blu, che tiene tra le zampe anteriori un disco solare rosso. Ai suoi lati vi sono due colonne di geroglifici neri su fondo giallo, bordate da due linee blu. Le altre due pareti presentano entrambe l'immagine del dio Anubi, raffigurato con il corpo mummificato rosso, il collare usekh giallo con filari neri, la testa di sciacallo nera e una parrucca tripartita blu. Il dio è rappresentato stante fra due colonne di geroglifici simili alle precedenti, nell'atto di impugnare con entrambe le mani un lembo di fazzoletto ripiegato.

Le iscrizioni recano il seguente testo:
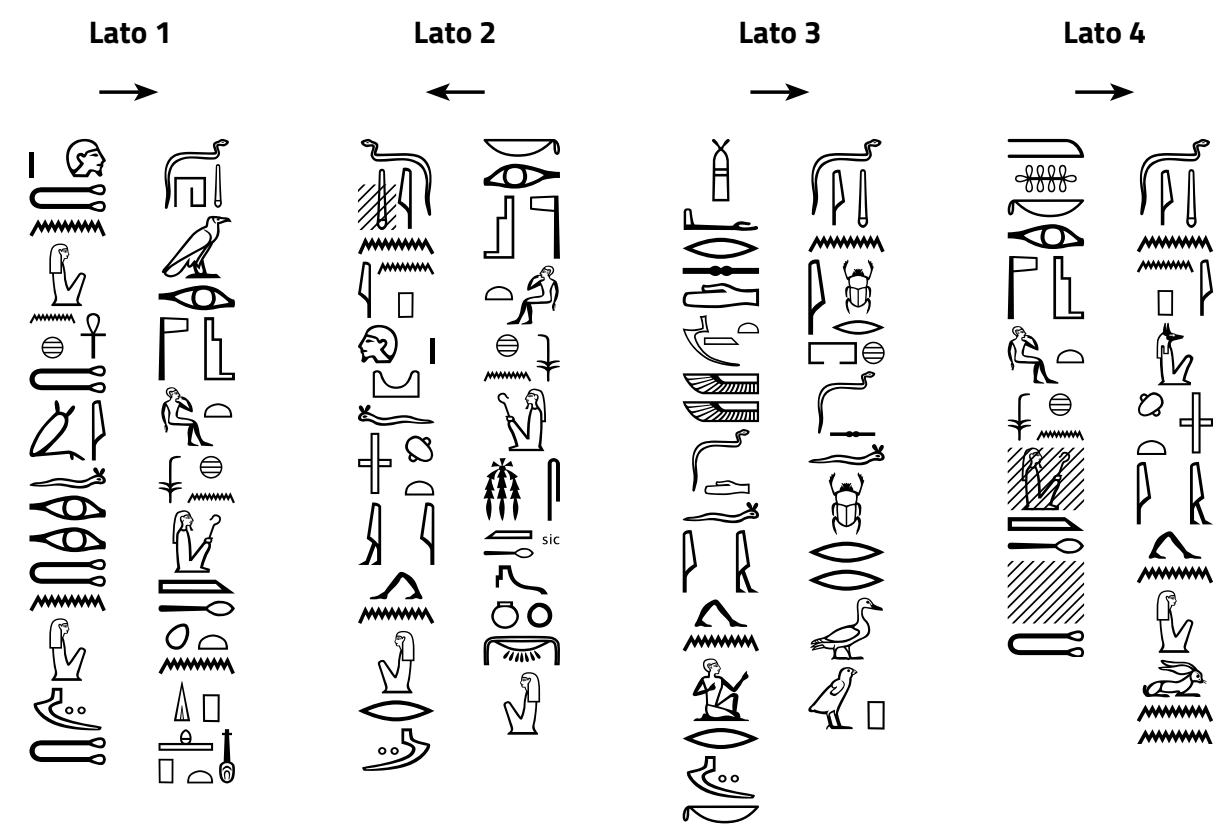

Lato 1:

dd md.w h3 Wsjr T3-šrj.t-(nt-)hns.w m3 ${ }^{e}(. t)$ hrw s3.t (n.t)

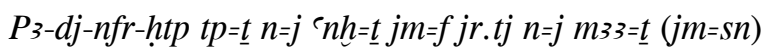
Parole dette: "Salve, l'Osiri Tasherit(net)khonsu, ${ }^{11}$ giusta di voce, figlia di Padineferhotep; la tua testa è per me, affinché tu viva attraverso di essa; i tuoi due occhi sono per me, affinché tu veda (attraverso di essi).

Lato 2:

$\underline{d d}$ md.w jn Jnp.w tp.y $\underline{d} w=f j m . j$ wt jj. $n=j \quad r \quad m 33=k$ Wsjr

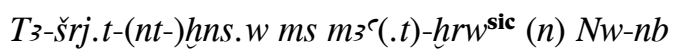

Parole dette da Anubi, colui che è sulla sua montagna, colui che è nel luogo dell'imbalsamazione: "Sono venuto per vedere te, l'Osiri Tasherit(net) khonsu, giusta di voce, nata da Nuneb" ${ }^{12}$
Lato 3:

$\underline{d} d$ md.w jn Hpr.j hpr $\underline{d} s=f^{13} h p r r^{14} p w \subset p r\{s\}^{15} d m 3 . t y$ $\underline{d} d=f j j . n=j \quad r \quad m 33=k$

Parole dette da Khepri, colui che si genera da sé, (egli) è lo scarabeo provvisto di ali. Egli dice: "Io sono venuto per vederti”.

Lato 4:

$\underline{d} d$ md.w jn Jnp.w jm.j wt jj.n.j wnn(=j) $m$ s3=k Wsjr

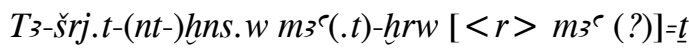

Parole dette da Anubi, colui che è nel luogo dell'imbalsamazione: "Sono venuto per essere la tua protezione, l’Osiri Tasherit(net)khonsu, giusta di voce, $[<$ per $>\operatorname{veder}($ ?) $]$ ti. 


\section{Datazione}

Come precisato sopra, il contesto di provenienza delle due cassette è ignoto. Inoltre le due proprietarie Tabes e Tasheritnetkhonsu - non sono personaggi noti alla letteratura egittologica. Pertanto la datazione può essere suggerita solo dallo stile della decorazione, che trova dei confronti molto convincenti con le cassette di Mutirdis (BM EA 8531), ${ }^{16}$ di Asetemakhbit (BM EA 8532) ${ }^{17}$ e di Padiamunnebnesuttauy VI (BM EA 8533), ${ }^{18}$ che hanno dimensioni molto simili, la forma pressoché cubica, il fondo chiaro, le immagini raffiguranti Anubi mummiforme, le due colonne di geroglifici neri su fondo giallo e la banda rossa lungo gli spigoli su ogni singola parete.

Marie Cécile Bruwier, sulla base dell'analisi stilistica, le data non prima della XXX dinastia, ${ }^{19}$ supposizione confermata da evidenze archeologiche che datano i contesti di provenienza delle cassette di questo tipo fra la XXX dinastia e l'epoca romana. ${ }^{20}$

Inoltre Padiamunnebnesuttauy e Asetemakhbit, rispettivamente proprietari delle cassette $\mathrm{BM}$ EA 8533 e BM EA 8532, sono membri delle famiglie di Djedkhonsuiuefankh e Mutmin, le quali all'inizio dell'età tolemaica avevano occupato alcune camere della tomba di Epoca Tarda di Ankhhor nell'Asasif (TT 414). ${ }^{21} \mathrm{Ne}$ consegue che quasi certamente anche le cassette torinesi si possono datare in una fase iniziale dell'età tolemaica. Supporta questa datazione anche il calzante confronto tra la cassetta di Tasheritnetkhonsu (Torino Cat. 2425) e i frammenti di una cassetta lignea (Vienna A 1993) ${ }^{22}$ ritrovati negli strati tolemaici della tomba VII dell'Asasif, pubblicata da Julia Budka. ${ }^{23}$ In particolar modo, della cassetta originaria si conserva quasi integralmente una parete dove uno scarabeo con disco solare tra le zampe anteriori (il dio Khepri) è rappresentato tra due colonne di geroglifici neri su fondo giallo. ${ }^{24}$ Un altro frammento di parete conserva la rappresentazione del dio Anubi mummificato a testa di sciacallo. Infine, il coperchio presenta una colonna di geroglifici sulla quale è rappresentato, specularmente, uno sciacallo accovacciato con flabello tra le zampe anteriori. $^{25}$

\section{Una breve prosopografia}

Sulla cassetta Torino Cat. 2423 sono citati i genitori di Tabes, Padiamunnebnesuttauy e Mutirdis.
Entrambi sono nomi largamente attestati in Epoca Tarda e all'inizio del periodo tolemaico. ${ }^{26}$ Inoltre il nome Padiamunnebnesuttauy ricorre con particolare frequenza nelle suddette famiglie di Djedkhonsuiuefankh e Mutmin occupanti la tomba di Ankhhor. Al British Museum si conserva la cassetta per viscere EA 8531, appartenente ad una certa Mutirdis, madre del Sacerdote di Amon-Ra presso il Tempio di Karnak Padiamunnebnesuttauy, di cui si conserva anche una stele in una collezione privata a Colonia. ${ }^{27}$ Non possiamo certamente sostenere che si tratti della stessa persona; tuttavia i nomi, la somiglianza stilistica tra le cassette e la datazione non solo suggeriscono l'appartenenza di Tabes alla stessa famiglia della Mutirdis proprietaria della cassetta BM EA 8531 e della stele, ma un legame diretto con le famiglie di Djedkhonsuiuefankh e Mutmin, se non addirittura un'appartenenza alle stesse.

Il nome della proprietaria della cassetta Torino Cat. 2425, Tasheritnetkhonsu, è attestato in Epoca Tar$\mathrm{da}^{28} \mathrm{e}$ in età tolemaica. Anche in questo caso si riscontra un'omonimia tra i membri delle famiglie tolemaiche che occuparono la tomba di Ankhhor. ${ }^{29}$ Non si tratta però certamente dello stesso personaggio poiché la Tasheritnetkhonsu della TT 414 è figlia di Paiuhor, ${ }^{30}$ mentre la proprietaria della cassetta Torino Cat. 2425 è figlia di Padineferhotep.

\section{Funzione}

Le cassette Torino Cat. 2423 e 2425 giunsero al Museo Egizio prive del loro contenuto, tanto che, come abbiamo visto, furono chiamate da Orcurti, da Fabretti et al. semplicemente "cassette funerarie". Esse, tuttavia, presentano una decorazione che riconduce immediatamente alle cassette per contenere le viscere, ${ }^{31}$ sulle quali erano sovente rappresentati gli dei Anubi, Sokar e Khepri. ${ }^{32}$

Anubi è il dio ritratto più frequentemente, ${ }^{33}$ di solito con i seguenti epiteti: $n b$ tz-dsr "signore della terra sacra"; tpy $\underline{d} w=f$ "colui che risiede sulla sua montagna"; jmy wt "colui che risiede nel luogo dell'imbalsamazione"; hnty sḥ-ntrr "il primo del padiglione divino"; ${ }^{34}$ e $n b$ krr.t "signore della caverna".

Sulla cassetta Cat. 2423 è, appunto, $n b$ krr.t e tpy $\underline{d} w=f$, mentre sulla cassetta Cat. 2425 è tpy $\underline{d} w=f e$ jmy $w t$. Insieme a Sokar, che è raffigurato sulla cassetta Cat. 2425, oltre a essere una divinità particolarmen- 
te connessa alla rigenerazione delle viscere e alla loro vitalità, permette di ristabilire i cinque sensi del defunto. ${ }^{35}$ In particolar modo, in numerosi Libri dei Morti, Sokar - o la divinità sincretica Sokar-Osiri - è rappresentato seduto o stante davanti a una cassa canopica sormontata dal falco. ${ }^{36}$

Un ulteriore elemento riconducibile al concetto di rigenerazione, possibile solo grazie al completo funzionamento del corpo del defunto, mediante il rinvigorimento degli organi eviscerati è costituito dalla presenza di Khepri, rappresentato sulla cassetta Torino Cat. 2425 su un santuario, con il disco solare, simbolo del concetto di ciclicità per eccellenza, tra le zampe anteriori. Il programma decorativo non lascia pertanto dubbi sul fatto che le due cassette fossero originariamente destinate alla preservazione delle viscere. C'è da dire, tuttavia, che esse non presentano alcuna traccia di residui organici sulle pareti interne e il dato risulta singolare se si considera che dalla XXX dinastia l'uso dei vasi canopi non è più attestato. ${ }^{37}$

\section{Conclusioni}

Le cassette di Tabes (Cat. 2423) e Tasheritnetkhonsu (Cat. 2425) si datano inequivocabilmente tra la XXX dinastia e l'inizio dell'età tolemaica. Inoltre, abbiamo già osservato come sulla cassetta di Tabes sia scritto il nome del padre Padiamunnebnesuttauy, ${ }^{38}$ lo stesso portato da diversi membri della famiglia di Djedkhonsuiuefankh, ${ }^{39}$ mentre un'omonima di Tasheritnetkhonsu fu moglie di Hor.

Se si tiene conto del suo stile, così simile a quello delle cassette BM EA 8531, BM EA 8532, BM EA 8533 e Vienna A 2126, tutte provenienti dalle sepolture tolemaiche della tomba di Ankhhor (TT 414), ${ }^{40}$ possiamo ipotizzare che il padre di Tabes e Hor, quindi Tabes e Tasheritnetkhonsu, appartengano alle stesse famiglie e che quindi le due cassette provengano dallo stesso contesto. È interessante notare che al Museo Egizio sono presenti numerosi oggetti provenienti dalle sepolture tolemaiche della TT 414, arrivati con la collezione Drovetti. ${ }^{41}$

\begin{tabular}{|l|l|l|l|}
\hline Collezione & Oggetti & $\begin{array}{l}\text { Famiglie di } \\
\text { Djedkhonsuiuefankh } \\
\text { e Mutmin }\end{array}$ & Famiglie di Hor e Tasheritnetkhonsu \\
\hline \multirow{5}{*}{ Drovetti } & Casetta di Tabes (Cat. 2423) & $\begin{array}{l}\text { Figlia di } \\
\text { Padiamunnebnesuttauy (?) }\end{array}$ & \\
\cline { 2 - 4 } & $\begin{array}{l}\text { Stele di Neskhonsu I (Cat. } \\
\text { 1597) }\end{array}$ & $\begin{array}{l}\text { Figlia di } \\
\text { Padiamunnebnesuttauy I }\end{array}$ & \\
\cline { 2 - 5 } & $\begin{array}{l}\text { Stele di Padiamunnebnesuttauy } \\
\text { V (Cat. 1573) }\end{array}$ & Figlio di Mutmin & \\
\cline { 2 - 4 } & $\begin{array}{l}\text { Libro dei Morti Torino di Djedhor } \\
\text { (Cat. 1830) }\end{array}$ & $\begin{array}{l}\text { Fratello di } \\
\text { Djedkhonsuiuefankh }\end{array}$ & \\
\cline { 2 - 4 } & $\begin{array}{l}\text { Cassetta per viscere di } \\
\text { Asetemakhbit II (Cat. 2426) }\end{array}$ & $\begin{array}{l}\text { Figlia di Pakherenkhonsu e } \\
\text { Neskhonsu }\end{array}$ & \\
\cline { 2 - 5 } & $\begin{array}{l}\text { Cassetta di Tasherinetkhonsu } \\
\text { (Cat. 2425) }\end{array}$ & - & figlia di Padineferhotep (?) e di Nuneb (?) \\
\cline { 2 - 4 } & Stele di Horkhonsu (Cat. 1614) & - & Figlio di Tasheritnetkhonsu (?) \\
\hline
\end{tabular}

Tab. 1: Oggetti conservati al Museo Egizio, provenienti dalle sepolture tolemaiche della TT 414.

Sebbene il programma decorativo e i testi delle cassette Torino Cat. 2423 e Cat. 2425 confermino che si doveva trattare di contenitori prodotti per le viscere della mummia, come abbiamo visto essi sono totalmente privi di residui organici. Non si tratta di un caso isolato: anche la maggior parte delle cassette contemporanee non presentano tracce di residui organici o di elementi riconducibili alla collocazione di pacchetti con organi mummificati. Inoltre le uniche cassette per viscere di questo periodo che hanno restituito vere e proprie viscere fino a oggi sono una cassetta ritrovata nella tomba 50 del cimitero G di Abido, datata da Petrie intorno alla XXX dinastia, che conteneva viscere bendate, ${ }^{46}$ e un'altra menzionata da D'Athanasi. ${ }^{47}$ Questo dato in effetti concorda con quanto sappiamo circa il cambiamento delle usanze 
funerarie attestato tra la fine dell'Epoca Tarda e l'inizio dell'età tolemaica: ${ }^{48}$ in questo periodo fu mantenuta la consuetudine di mummificare i corpi, ma l'eviscerazione non fu più ritenuta fondamentale. ${ }^{49}$ Infatti sono attestate mummie non eviscerate oppure con gli organi trattati e ricollocati all'interno della cavità addominale, accompagnate da corredi funerari e comunque "cassette per viscere" che, più correttamente, sono da interpretare come dei simulacri, ossia il riflesso sbiadito di un'usanza obsoleta. ${ }^{50}$ Tale considerazione non mette di certo in dubbio la funzione delle cassette in questione, ma pone il problema della rifunzionalizzazione degli oggetti del corredo funerario, attestata con particolare frequenza dall'Epoca Tarda in poi.

I frutti di palma dum e i melograni ritrovati dentro le cassette per viscere di Mutirdis ${ }^{51}$ (Londra EA 8531) e di Asetemakhbit (Londra EA 8532) sono stati ivi deposti per una decisione presa al momento o per un gesto premeditato che di fatto regala ai due contenitori una doppia funzione (una pratica e una simbolica) $?^{52}$

La seconda ipotesi potrebbe essere avvalorata dalle due cassette "per viscere" appartenute ad un certo Djedhor, datate alla XXX dinastia, provenienti dalla tomba 50 della necropoli G di Abido. Esse furono infatti ritrovate da Petrie con all'interno 394 ushabti $^{53}$ (rispettivamente 196 e 198), un totale che si avvicina

\section{Note}

${ }^{1} \mathrm{Mi}$ è molto gradito ringraziare per la disponibilità e per i preziosi suggerimenti Marie Cécile Bruwier, Andrea Fanciulli e Federico Poole.

${ }^{2}$ Direttore del Regio Museo Egizio dal 1861 al 1870.

${ }^{3}$ Orcurti, Catalogo illustrato, 1855, p. 114.

${ }^{4}$ Fabretti et al., Regio Museo di Torino, 1882, pp. 340-41.

${ }^{5}$ Le due cassette non si individuano nemmeno nella lista stilata per la vendita degli oggetti della collezione del Console e consegnata a Carlo Vidua. Cfr. Documenti inediti, 1880, pp. 206-93.

${ }^{6}$ Per la storia della nascita del Museo Egizio e per la bibliografia principale, v. Moiso, La storia del Museo Egizio, 2016; Moiso, in Museo Egizio, 2015, pp. 20-35.

${ }^{7} L G G$, I, p. 397.

${ }^{8} L G G$, III, p. 760.

${ }^{9}$ La lettura si fa incerta dopo il monolittero "s". Il senso stesso di questo segno è dubbio: si tratta forse del complemento fonetico di $m s$, usato per sostituire l'intera parola a causa della mancanza di spazio. Per lo stesso problema non scrive il nome completo " $M w . t-j r$ $d j-s$ ", ma soltanto i segni $d j$ e s seguito dal segno $m_{3}{ }^{e}$ ai canonici 401 ushabti previsti per singola sepoltura, a partire dal Terzo Periodo Intermedio. ${ }^{54}$ Una suddivisione così oculata, a mio avviso, rende meno probabile che si sia trattata della risposta ad un'esigenza manifestatasi al momento dell'assemblaggio e deposizione del corredo funerario; ${ }^{55}$ si potrebbe trattare invece di una disposizione che testimonia una interscambiabilità funzionale o addirittura di una "doppia funzione" delle cassette funerarie di questo periodo. Questo spiegherebbe, ad esempio, perché le cassette per canopi e per viscere, in passato e talvolta ancora oggi, siano scambiate per cassette per ushabti $^{56}$ e viceversa. $^{57}$

Purtroppo ad oggi non è possibile confermare questa ipotesi, sebbene la somiglianza morfologica e iconografica di oggetti come cassette canopiche e cassette per ushabti prodotte in Epoca Tarda potrebbe essere proprio una conseguenza di questo meccanismo. Innegabilmente, la mancanza di contesti archeologici intatti, in grado di restituirci una panoramica completa, attendibile e non sommaria dei corredi tombali, ${ }^{58}$ nonostante i numerosi studi di singole tipologie di oggetti del corredo funerario, ${ }^{59}$ costituisce una vera e propria falla. Pertanto, fino a quando non si avranno maggiori dati non potremo apprendere appieno taluni aspetti e meccanismi dei costumi funerari dell'Egitto della seconda metà del I millennio a.C.

per l'epiteto classico $m 3^{e}-h r w$.

${ }^{10} \mathrm{Si}$ tratta di costruzioni in materiali leggeri, molto semplici, di cui rimangono solo delle rappresentazioni. Essi dovevano essere costituiti da una camera fatta di mattoni crudi o, probabilmente, cannicciato, coperta da un soffitto del tipo a botte realizzato con canne e altri materiali vegetali. Cfr. Hayes, The Scepter of Egypt, 1959, II, p. 448.

${ }^{11}$ PN, I, p. 369, n. 19.

${ }^{12} P N$, I, p. 181 , n. 24.

${ }^{13} L G G$, V, p. 705.

${ }^{14} L G G$, V, pp. 718-19.

${ }^{15}$ Potrebbe trattarsi di un errore dello scriba che scambia il segno (s) con il determinativo (wi c c c pr. Cfr. Wb. Zettel 31.394.500: Abu Simbel (= LD, III, tav. 195a); Wb. Zettel 31.394.560: Edfu (= Rochemonteix e Chassinat, Le Temple d'Edfou, I.3, 1987, p. 381).

${ }^{16}$ Aston, ÄgLev 10 (2000), p. 162; British Museum collection online.

${ }^{17}$ Aston, $\ddot{A} g L e v 10$ (2000), p. 162; Reiser-Haslauer, in Bietak e Reiser-Haslauer, Das Grab des 'Anch-Hor, 1982, 
p. 268; https://www.britishmuseum.org/collection/ object/Y_EA8532.

${ }^{18}$ Budka et al., ÄgLev 22/23 (2012/2013), p. 239, fig. 22; https://www.britishmuseum.org/collection/object/Y_ EA8533.

${ }^{19}$ Bruwier, "Les coffrets à viscères humains", 1990. Non è invece plausibile la data tra la XXI e la XXVI dinastia proposta da Aston, ÄgLev 10 (2000), pp. 162-63.

${ }^{20}$ Cfr. Kowalska et al., in Myśliwiec, Saqqara III, 2008, pp. 335-43; Bridonneau e Lecuyot, Archaeologia 445 (2007), p. 46; Lecuyot, Archaeologia 413 (2004), pp. 29-30.

${ }^{21}$ Cfr. Budka et al., ÄgLev 22/23 (2012/2013), p. 237; Kampp, Die Thebanischen Nekropole, I, 1996, p. 614; Bietak e Reiser-Haslauer, Das Grab des 'Anch-Hor, I-II, 1978-1982.

${ }^{22}$ Cfr. Budka, Bestattungsbrauchtum und Friedhofsstruktur im Asasif, 2010, pp. 620-21, tav. 32, a, b.

${ }^{23}$ Budka, Bestattungsbrauchtum und Friedhofsstruktur im Asasif, 2010, pp. 278-80.

${ }^{24} \mathrm{Cfr}$. con il lato 3 della cassetta Cat. 2425, infra, tav. VII.

${ }^{25} \mathrm{~V}$. Budka, Bestattungsbrauchtum und Friedhofsstruktur im Asasif, 2010, fig. 126.

${ }^{26}$ Vittmann, Priester und Beamte im Theben der Spätzeit, 1978, pp. 61-100.

${ }^{27}$ Acquistata da un collezionista di Colonia nel 1965 e pubblicata nel 1976 da Doetsch-Amberger, GM 19 (1976), pp. 23-27.

${ }^{28}$ PN, I, 1935, p. 369, n. 19.

${ }^{29}$ Bietak, in Bietak e Reiser-Haslauer, Das Grab des 'Anch-Hor, II, 1982, p. 256.

${ }^{30} \mathrm{Cfr}$. l'albero genealogico in Bietak e Reiser-Haslauer, Das Grab des 'Anch-Hor, II, 1982, p. 256.

${ }^{31}$ Importanti studi sull'evoluzione tipologica sono: Aston, ÄgLev 10 (2000), pp. 159-78; Dodson, The Canopic Equipment, 1994; Lüscher, Untersuchungen zu ägyptischen Kanopenkästen, 1990; Dobrowolska, ET 4 (1970), pp. 73-85.

${ }^{32}$ Per l'iconografia delle cassette per viscere di età tolemaica v. Bruwier, Cahiers de Mariemont 30-31 (2003), pp. 18-38; Bruwier, "Les coffrets à viscères humains", 1990; Bruwier, Cahiers de Mariemont 13 (1982), pp. 4-21.

${ }^{33}$ Bruwier, in Clarysse et al. (a cura di), Egyptian Religion, 1998, pp. 76-79.

${ }^{34}$ Bruwier, in Clarysse et al. (a cura di), Egyptian Religion, 1998, p. 77.

${ }^{35}$ Budka et al., ÄgLev 22/23 (2012/2013), pp. 240-42.

${ }^{36}$ Budka et al., ÄgLev 22/23 (2012/2013), p. 241.

${ }^{37}$ Cfr. Ikram, Death and Burial, 2015, pp. 68-73; Taylor, Death and the Afterlife, 2001, pp. 86-87; Ikram e Dodson, The Mummy in Ancient Egypt, 1998, pp. 160-65.

${ }^{38} \mathrm{PN}$, I, 1935, p. 122, 6.

${ }^{39} \mathrm{Cfr}$. albero genealogico della famiglia di Djedkhonsuiuefankh in Budka et al., ÄgLev 22/23 (2012/2013), p. 211.

${ }^{40}$ Anche la cassetta per viscere di Asetemakhbit (Torino Cat. 2426), conservata al Museo Egizio di Torino, potrebbe provenire dalla TT 414. V. Budka et al., ÄgLev 22/23 (2012/2013), p. 234, nota 127.

${ }^{41}$ Roccati, in Morigi Govi et al. (a cura di), L'Egitto fuori
dell'Egitto, 1991, p. 360.

${ }^{42}$ Bietak, in Bietak e Reiser-Haslauer, Das Grab des 'Anch-Hor, II, 1982, p. 252; Munro, Die spätägyptischen Totenstelen, 1973, pp. 56-57, 234.

${ }^{43}$ Bietak, in Bietak e Reiser-Haslauer, Das Grab des 'AnchHor, II, 1982, p. 254; Munro, Die spätägyptischen Totenstelen, 1973, p. 237.

${ }^{44}$ Bietak e Reiser-Haslauer, Das Grab des 'Anch-Hor, II, 1982, p. 252.

${ }^{45}$ Roccati, in Morigi Govi et al. (a cura di), L'Egitto fuori dell'Egitto, 1991, p. 360.

${ }^{46}$ Cfr. Petrie, Abydos, I, 1902, p. 39.

${ }^{47}$ Cfr. D’Athanasi, A Brief Account of the Researches, 1936, pp. 117-18.

${ }^{48}$ Sull'argomento si veda Smith, Following Osiris, 2017, pp. 358-60; Smith, Traversing Eternity, 2009, pp. 30-49.

${ }^{49}$ Smith, Traversing Eternity, 2009, p. 31.

${ }^{50}$ Tra le tombe di Abydos, scavate da Petrie, una conservava delle cassette con all'interno impacchi costituiti da bende di lino e cocci (cfr. Petrie, Abydos, I, 1902, p. 54); così come la cassetta di Hornedief (Leiden AH 215), datata tra il regno di Tolomeo II e III, contenente impacchi di bende e cocci (Petrie, Abydos, I, 1902, p. 167). Entrambe sono il chiaro riflesso di un'usanza oramai superata che persiste nella forma dell'evocazione ed emulazione.

${ }^{51}$ Aston, $\ddot{A} g L e v 10$ (2000), p. 163.

${ }^{52}$ Ammesso che costituiscano il contenuto originario e non il frutto di un'azione moderna, ossia ottocentesca.

${ }^{53}$ Petrie, Abydos, I, 1902, p. 38.

${ }^{54}$ Schneider, Shabtis, I, 1977, p. 320.

${ }^{55}$ Aston aveva sminuito il dato giustificandolo con la necessità da parte degli antichi egiziani di trovare un contenitore per trasportare le numerose statuette funerarie all'interno della tomba (Aston, ÄgLev 10 [2000], p.171).

${ }^{56} \mathrm{Nel}$ catalogo online del British Museum di Londra (https://www.britishmuseum.org/research/collection_ online/search.aspx) le cassette per viscere EA 43433 ed EA 35762 sono chiamate "shabti-box"; come anche la cassetta per viscere E.10.1887 nel catalogo online del Fitzwilliam Museum di Cambridge (https://www. fitzmuseum.cam.ac.uk/).

${ }^{57}$ Il caso più emblematico è la cassetta per ushabti di Nia (Torino Cat. 2444), scambiata per una cassetta canopica da Habachi, BIFAO 71 (1972), p. 77.

${ }^{58}$ V. Khótay, Burial and Mortuary Practices, 2017; Aston, in Strudwick e Taylor (a cura di), The Theban Necropolis, 2003, pp. 138-66.

${ }^{59}$ Marini, EVO 35 (2012), pp. 83-124. Aston, ÄgLev 10 (2000), pp. 159-78; Aston, OMRO 74 (1994), pp. 21-54; Taylor, Egyptian Coffins, 1989; Silvano, EVO 3 (1980), pp. 83-97; Raven, OMRO 50-60 (1978-1979), pp. 251-96; Munro, Die spätägyptischen Totenstelen, 1973; Reisner, Canopics, 1967.

\section{Bibliografia}

Aston, D.A., "Canopic Chests from the Twenty-first Dynasty to the Ptolemaic Period”, ÄgLev 10 (2000), pp. 159-78.

Aston, D.A., “The Shabti-Box: A Typological Study”, OMRO 
74 (1994), pp. 21-54.

Aston, D.A., “The Theban West Bank from the Twentyfifth Dynasty to the Ptolemaic Period", in: N.

Strudwick e J. H. Taylor (a cura di), The Theban Necropolis: Past, Present and Future, London 2003, pp. 138-66.

Bietak, M., "Die Reliefkunst im Grab des 'Anch-Hor", in

M. Bietak e E. Reiser-Haslauer, Das Grab des 'Anch-Hor, Obersthofmeister der Gottesgemahlin Nitokris II (UZK 5), Wien 1982, pp. 221-57.

Bietak, M. e E. Reiser-Haslauer, Das Grab des 'Anch-Hor, Obersthofmeister der Gottesgemahlin Nitokris I (UZK 4), Wien 1978.

Bietak, M. e E. Reiser-Haslauer, Das Grab des 'Anch-Hor, Obersthofmeister der Gottesgemahlin Nitokris II (UZK 5), Wien 1982.

Bridonneau, C. e G. Lecuyot, “Saqqara à la Basse Époque”, Archéologia 445 (2007), pp. 34-46.

Bruwier, M.C., "Enquête sur un coffret égyptien d'époque ptolémaïque”, Cahiers de Mariemont 13 (1982), pp. 4-21.

Bruwier, M.C., "Les coffrets à reliques funéraires, Anubis et les Enfants d'Horus", Cahiers de Mariemont 30-31 (2003), pp. 18-38.

Bruwier, M.C., "Les coffrets à viscères humains en Égypte (de la $30^{e}$ dynastie à l'époque romaine)" (tesi di dottorato, Université Catholique de Louvain), Louvain 1990.

Bruwier, M.C., "Présence et action d'Anubis sur le coffret d'un prêtre héracléopolitain”, in: W. Clarysse, A. Schoors e H. Willems (a cura di), Egyptian Religion: The Last Thousand Years. Studies Dedicated to the Memory of Jan Quaegebeur. Part I (OLA 84), Leuven 1998, pp. 63-79.

Budka, J., Bestattungsbrauchtum und Friedhofsstruktur im Asasif: eine Untersuchung der spätzeitlichen Befunde anhand der Ergebnisse der österreichischen Ausgrabungen in den Jahren 1969-1977 (UZK 34), Wien 2010.

Budka, J., T. Mekis e M.C. Bruwier, "Reuse of Saite Temple Tombs in the Asasif During the Early Ptolemaic Time: The Tomb Group of Mw.t-Mnw from TT 414", ÄgLev 22/23 (2012/2013), pp. 209-51.

"Collezione Drovetti, a. 1822. Catalogue de la collect. d'antiq. de mons. le chev. Drovetti”, in: Documenti inediti per servire alla storia dei musei d'Italia, III, Firenze - Roma 1880, pp. 206-92.

D'Athanasi, G., A Brief Account of the Researches and Discoveries in Upper Egypt Made Under the Direction of Henry Salt, Esq, London 1936.

De Meulenaere, H., "Notes de prosopographie thébaine. Quatrième série”, CdE 64 (1989), pp. 55-73.

Dobrowolska, K., "Genèse et évolution des boîtes à vasescanopes”, ET 4 (1970), pp. 73-85.

Dodson, A., The Canopic Equipment of the Kings of Egypt, London 1994.

Doetsch-Amberger, E., “Eine Stele der Mwt-jr-dj-s”, GM 19 (1976), pp. 23-27.

Fabretti, A., F. Rossi e R.V. Lanzone, Regio Museo di Torino. Antichità Egizie (Catalogo generale dei musei di antichità e degli oggetti d'arte raccolti nelle gallerie e biblioteche del regno, Serie Prima Piemonte, 1), I, Torino 1882.
Habachi, L., "Nia, the Wab-Priest and Doorkeeper of Amun-of-the-Hearing-Ear”, BIFAO 71 (1972), pp. 67-85.

Hayes, W.C., The Scepter of Egypt: A Background for the Study of the Egyptian Antiquities in the Metropolitan Museum of Art. The Hyksos Period and the New Kingdom (1675-1080 B.C.), II, New York 1959.

Ikram, S., Death and Burial in Ancient Egypt, Cairo-New York 2015.

Ikram, S. e A. Dodson, The Mummy in Ancient Egypt. Equipping the Dead for Eternity, London 1998.

Kampp, F., Die thebanischen Nekropole: zum Wandel des Grabgedankens von der XVIII. bis zur XX. Dynastie, I (Theben 13), Mainz am Rhein 1996.

Khótay, K.A. (a cura di), Burial and Mortuary Practices in Late Period and Graeco-Roman Egypt, Budapest 2017.

Kowalska, A., M. Radomska, M. Kaczmarek, K.O. Kuraszkiewicz e E. Kopp, “Catalogue of Burials”, in: K. Myśliwiec (a cura di), Saqqara III. The Upper Necropolis. Part I: The Catalogue with Drawings, Warsaw 2008, pp. 29-382.

L $\ddot{A}=$ Lexikon der Ägyptologie, I-VII, Wiesbaden 1975-1992.

Lecuyot, G., "Les momies découvertes à Saqqara", Archéologia 413 (2004), pp. 26-32.

LD = Lepsius, K.R., Denkmäler aus Aegypten und Aethiopien, Text 3. B. Theben, III, Leipzig 1900.

LGG = Leitz, C., Lexikon der ägyptischen Götter und Götterbezeichnungen, I-VII (OLA 110-116), Leuven 2002-2003.

Lüscher, B., Untersuchungen zu ägyptischen Kanopenkästen: vom Alten Reich bis zum Ende der Zweiten Zwischenzeit (HÄB 31), Hildesheim 1990.

Marini, P., "I contenitori di ushabti dei Musei Italiani”, EVO 35 (2012), pp. 83-124.

Marini, P., "I contenitori di ushabti: funzioni e concezioni" (tesi di dottorato, Università di Pisa), Pisa 2016.

Moiso, B., La storia del Museo Egizio, Modena 2016.

Moiso, B., "Storia del Museo Egizio", in: Museo Egizio, Modena 2015, pp. 20-35.

Munro, P., Die spätägyptischen Totenstelen (ÄF 25), I-II, Glückstadt 1973.

Orcurti, P.C., Catalogo illustrato dei Monumenti Egizi del R. Museo di Torino compilato dal professore Pier Camillo Orcurti, II, Torino 1855.

Petrie, W.M.F., Abydos, I (EEF 22), London 1902. Petrie, W.M.F., Abydos, II (EEF 24), London 1903.

PN, I = Ranke, H., Die ägyptischen Personennamen, I, Glückstadt 1935.

Raven, M.J., "Papyrus-Sheaths and Ptah-Sokar-Osiris Statues”, OMRO 59-60 (1978-1979), pp. 251-96.

Reiser-Haslauer, E., "Familien aus der zweiten Belegungszeit des Grabes (ca. Zeit der 30. Dynastie und frühe Ptolemäerzeit)", in: M. Bietak e E. ReiserHaslauer, Das Grab des 'Anch-Hor, Obersthofmeister der Gottesgemahlin Nitokris II (UZK 5), Wien 1982, pp. 252-56.

Reisner, G.A., Catalogue général des antiquités égyptiennes

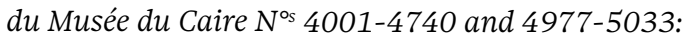
Canopics, Le Caire 1967.

Roccati, A., "La riscoperta continua dell'Egitto. "Preistoria" delle collezioni torinesi”, in: C. Morigi Govi, S. Curto e S. Pernigotti (a cura di), L'Egitto fuori dell'Egitto. Dalla 
riscoperta all'Egittologia, Bologna 1991, pp. 357-66.

Rochemonteix, M. [le Marquis de] e É. Chassinat, Le temple d'Edfou, I.3 (MIFAO 10), Le Caire $1987^{2}$.

Schneider, H.D., Shabtis: An Introduction to the History of Ancient Egyptian Funerary Statuettes with a Catalogue of the Collection of Shabtis in the National Museum of Antiquities at Leiden, I-III, Leiden 1977.

Silvano, F., "Le reticelle funerarie nell'antico Egitto: proposte di interpretazione”, EVO 3 (1980), pp. 83-97.

Smith, M., Following Osiris. Perspectives on the Osirian Afterlife from Four Millennia, Oxford 2017.
Smith, M., Traversing Eternity. Texts for the Afterlife from Ptolemaic and Roman Egypt, Oxford 2009.

Taylor, J.H., Egyptian Coffins (ShirEgypt 11), Aylesbury 1989. Taylor, J.H., Death and the Afterlife in Ancient Egypt, London 2001.

Vittmann, G., Priester und Beamte im Theben der Spätzeit: Genealogische und prosopographische Untersuchungen zum thebanischen Priester- und Beamtentum der 25. und 26. Dynastie (BeitrÄg 1), Wien 1978.

Ziegler, C., Les tombes hypogées de Basse Époque F7, F17, H, j1, Q, n1 (Fouilles du Louvre à Saqqara 2), Paris 2013. 

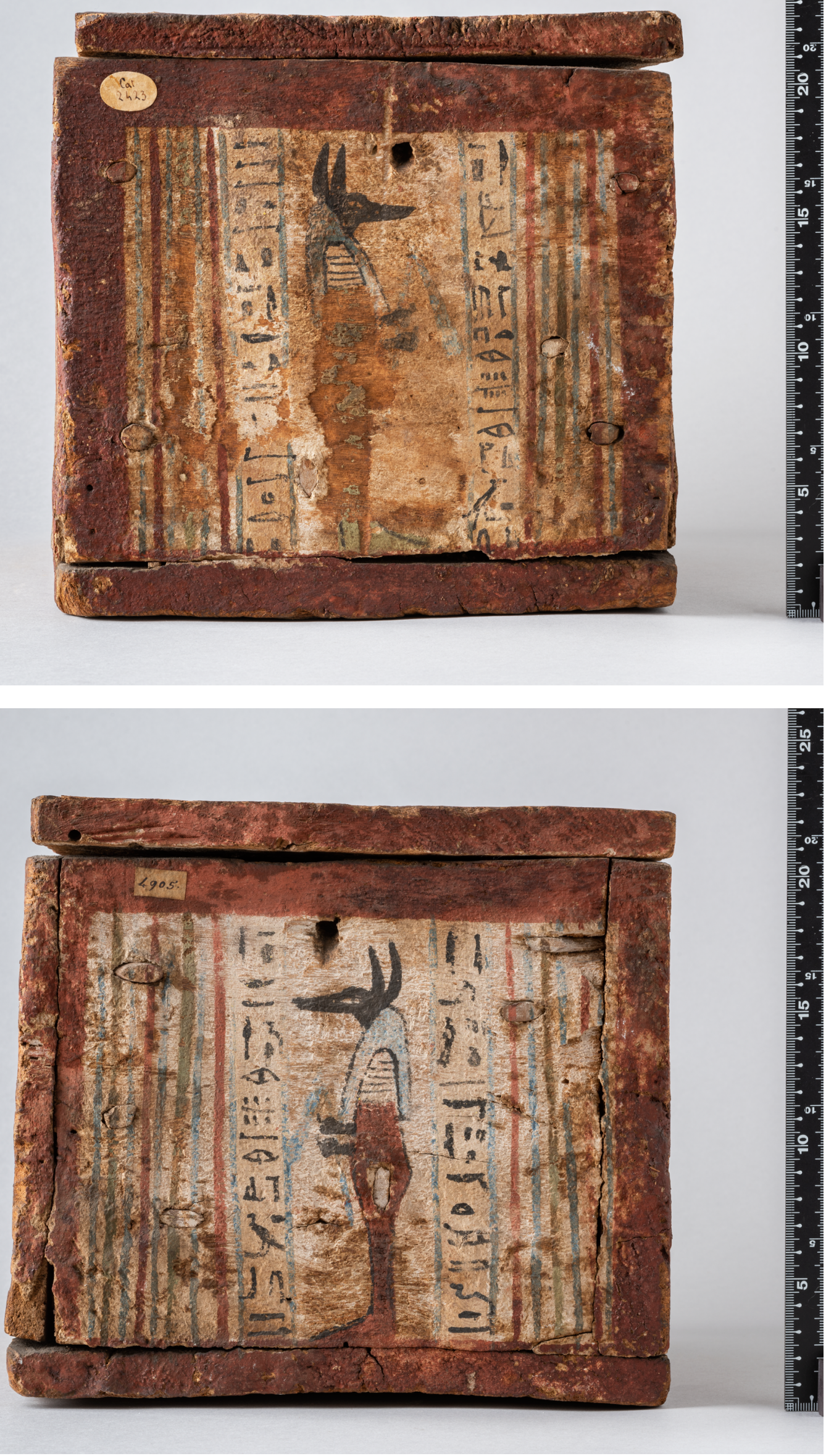

Tav. 1: Lati 1 (sopra) e 2 (sotto) della cassetta per viscere di Tabes (Torino Cat. 2423). H. 22,3 cm. Foto di Federico Taverni/ Museo Egizio, Torino. 

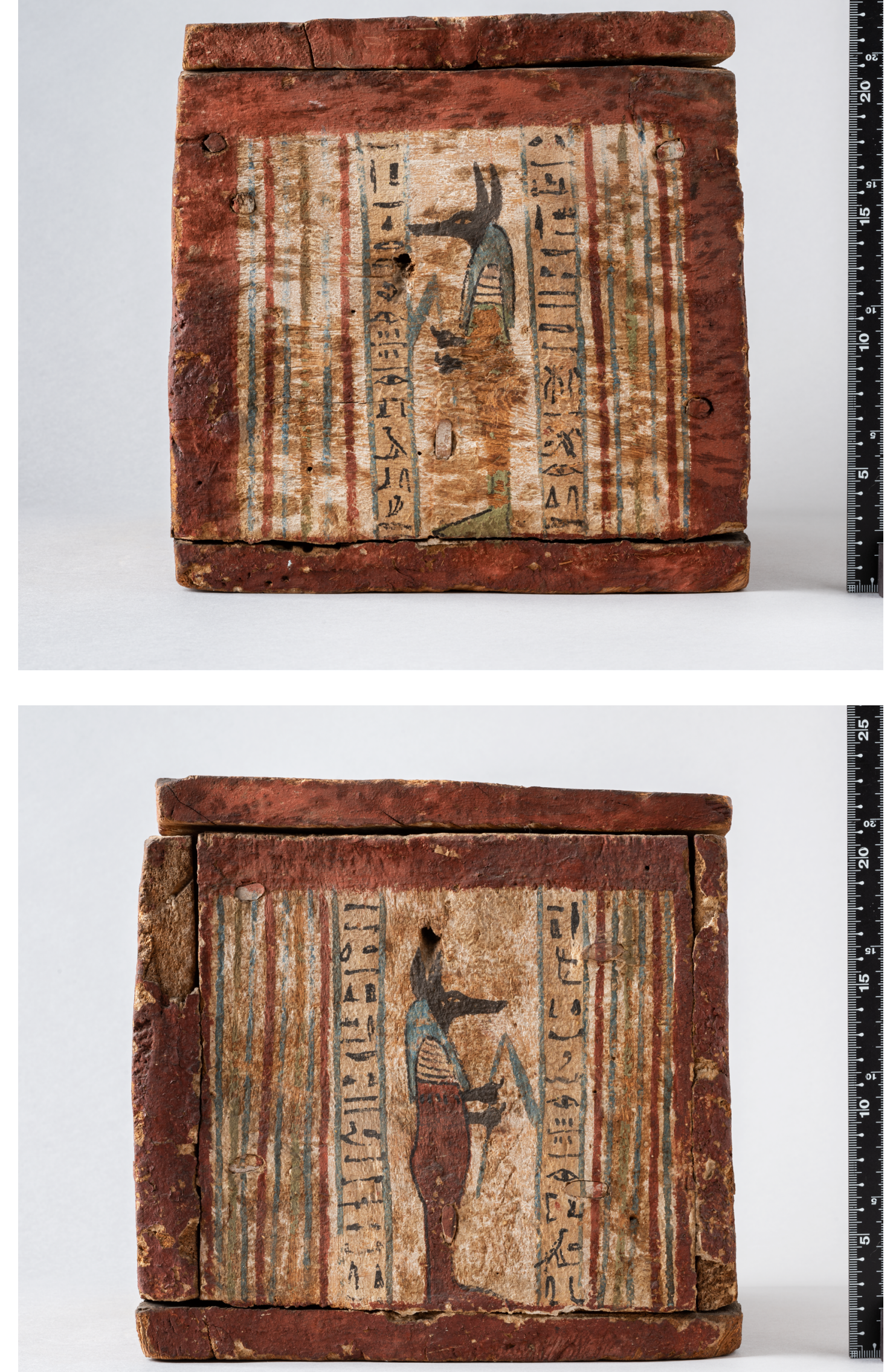

Tav. 2: Lati 3 (sopra) e 4 (sotto) della cassetta per viscere di Tabes (Torino Cat. 2423). H. 22,3 cm. Foto di Federico Taverni/ Museo Egizio, Torino. 


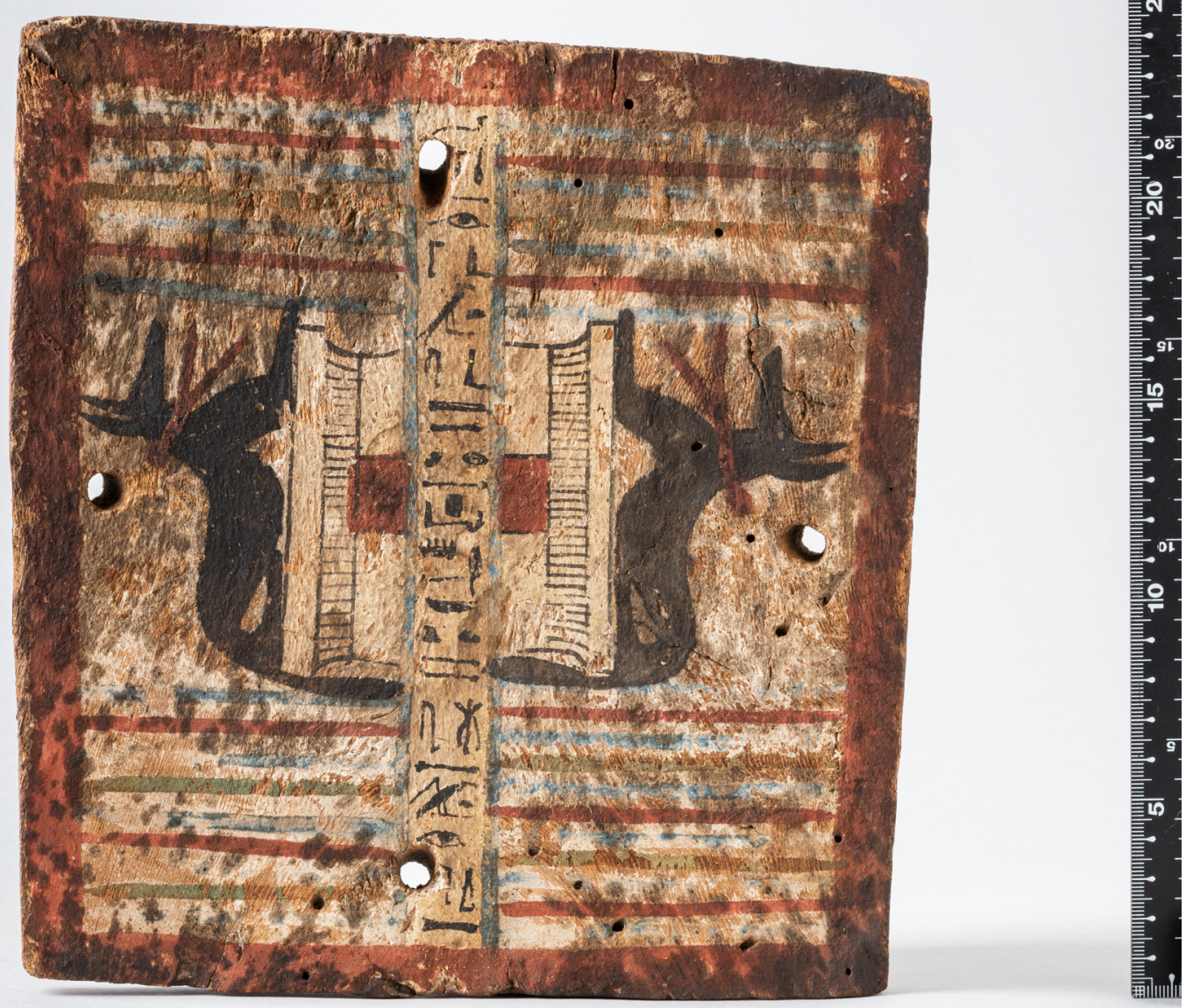

Tav. 3: Coperchio della cassetta per viscere di Tabes (Torino Cat. 2423). Foto di Federico Taverni/Museo Egizio, Torino. 

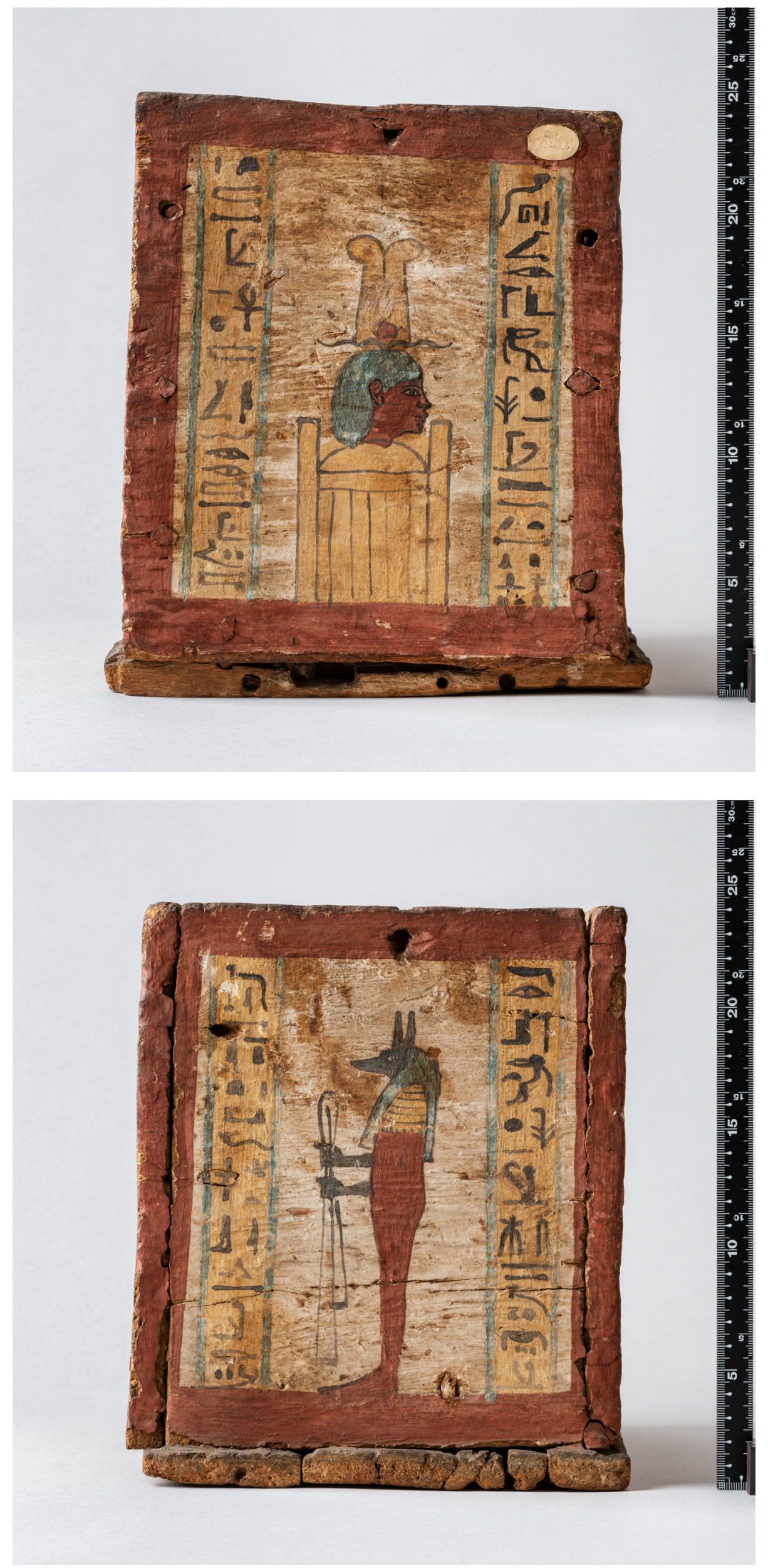

Tav. 4: Lati 1 (sopra) e 2 (sotto) della cassetta per viscere di Tasheritnetkhonsu (Torino Cat. 2425). H. 24,5 cm. Foto di Federico Taverni/Museo Egizio, Torino. 

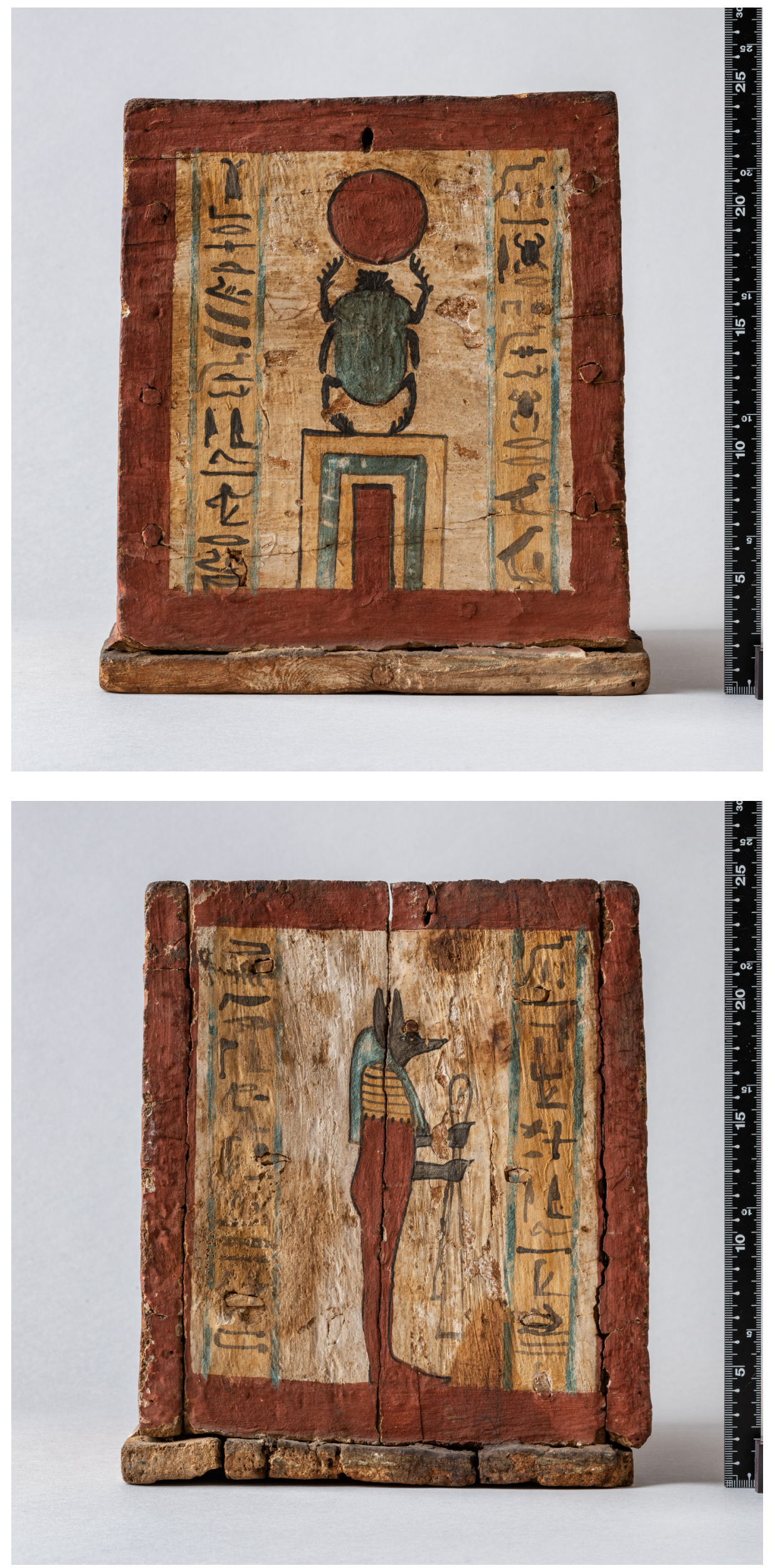

Tav. 5: Lati 3 (sopra) e 4 (sotto) della cassetta per viscere di Tasheritnetkhonsu (Torino Cat. 2425). H. 24,5 cm Foto di Federico Taverni/Museo Egizio, Torino. 